\title{
Synchronization in MB-OFDM-based UWB Systems
}

\author{
T. Jacobs ${ }^{1}$, Y. $\mathrm{Li}^{1}$, H. Minn ${ }^{1}$, and R.M.A.P. Rajatheva ${ }^{2}$ \\ ${ }^{1}$ Department of Electrical Engineering, University of Texas at Dallas \\ \{taj016000, yinghui.li, hlaing.minn $\} @$ utdallas.edu \\ ${ }^{2}$ Asian Institute of Technology, rajath@ait.ac.th.
}

\begin{abstract}
This paper presents a preamble-based low complexity synchronization method for MB-OFDM based UWB systems. The proposed synchronization method consists of sync detection, coarse timing estimation, fine timing estimation, and oscillator frequency offset estimation. The distinctive features of MBOFDM systems and the interplay between the timing and carrier frequency hopping at the receiver are judiciously incorporated in the proposed synchronization method. We also derive the probability density functions (pdfs) of the delays of the UWB channel paths which are used in optimizing our synchronization method. These pdfs are also useful in evaluating or optimizing the energy capture of other UWB systems. The effectiveness of our proposed synchronization method and optimization criteria are confirmed by computer simulation results.
\end{abstract}

\section{INTRODUCTION}

One of the promising ultra-wide band (UWB) technologies is multi-band orthogonal frequency division multiplexing (MB-OFDM) [1] which has been proposed for the IEEE 802.15.3a standard [3]. OFDM is a relatively mature technology and has been adopted in digital broadcasting, wireless LAN and MAN standards. OFDM has several advantages such as low complexity equalization in dispersive channels and the spectral scalability/adaptability. However, OFDM has some disadvantages such as larger susceptibility to nonlinear distortion at the transmit power amplifier [4] and larger sensitivity to synchronization errors [5]- [6]. The application of OFDM technology in UWB systems requires a high performance synchronization method, while UWB systems demand a low complexity approach.

There exist several preamble-based synchronization methods (see [7]- [14] and references therein) for regular OFDM systems. However, as recently reported in [15], MB-OFDM systems possess distinctive features which do not exist in regular OFDM systems. Utilizing these features, [15] presented best linear unbiased estimation (BLUE) based oscillator frequency offset (OFO) estimators for MB-OFDM systems.

In this paper, we present a synchronization method for MBOFDM systems based on the preamble of the IEEE 802.15.3a proposal. The proposed synchronization method consists of sync detection, coarse timing estimation, fine timing estimation and OFO estimation. In our synchronization design, we utilize the distinctive features of MB-OFDM systems and take into account the interplay between the timing and carrier frequency hopping at the receiver as well as complexity saving. We also derive the probability density functions (pdf) of the channel path delays of the IEEE 802.15 UWB channel model in [16]. Utilizing these pdfs, we present how to optimize the synchronization parameters. Note that these pdfs are also useful in evaluating or optimizing the energy capture of other UWB systems (e.g., [17]).

The rest of this paper is organized as follows. Section II describes the signal and channel models. The proposed synchronization method is presented in Section III and the synchronization parameter optimization is described in Section IV. Simulation results and discussions are provided in Section $\mathrm{V}$ and the paper is concluded in Section VI.

\section{Signal AND Channel Models}

In the MB-OFDM-based UWB system [3], the carrier frequency is hopped within a pre-defined set of carrier frequencies $\left\{f_{q}\right\}$ (corresponding to disjoint frequency bands) from symbol to symbol, according to a time-frequency code. The MB-OFDM system from [3] has 4 different preamble patterns (for 4 pico-nets), each associated with a different time-frequency code. Each preamble pattern is constructed by successively repeating a time-domain preamble sequence (symbol) over 21 periods. The preamble structure in each of the three different bands are shown in Fig. 1 for the pattern 1 (see [15] [3] for other patterns).

In the MB-OFDM-based system from [3], zero-padded guard intervals ( $N_{\text {pre }}$ prefix and $N_{\text {suf }}$ suffix zero samples; $N_{g}=N_{\text {pre }}+N_{\text {suf }} ; M_{0}=N+N_{g}$ ) are used instead of the conventional cyclic prefix guard interval. Denote the low-passequivalent time-domain samples (sampled at $N / T=1 / T_{s}, N$ times the sub-carrier spacing) transmitted in the $q$-th frequency band by $\left\{s_{q}(k)\right\}$. During the $m$-th symbol interval, i.e., $k \in\left[m M_{0}-N_{\text {pre }},(m+1) M_{0}-N_{\text {pre }}-1\right],\left\{s_{q}(k)\right\}$ can be all zeros if no signal is transmitted in the $q$-th band.

The IEEE 802.15.3a UWB RF channel model described in [16] is given by

$$
h_{\mathrm{RF}}(t)=X \sum_{l=0}^{L_{h}} \sum_{k=0}^{K} \alpha_{k, l} \delta\left(t-T_{l}-\tau_{k, l}\right)
$$

where $T_{l}, \tau_{k, l}$, and $X$ are random variables representing the delay of the $l$-th cluster, the delay (relative to the $l$-th cluster arrival time) of the $k$-th multipath component of the $l$-th cluster, and the log-normal shadowing, respectively. $\alpha_{k, l}$ are log-normal the multipath gain coefficients with

$$
E\left[\left|\alpha_{k, l}\left(T_{l}, \tau_{k, l}\right)\right|^{2}\right]=\Omega_{0} e^{-T_{l} / \Gamma} e^{-\tau_{k, l} / \gamma} .
$$


Details of the channel models are referred to in [16].

In this paper, we consider a low-pass-equivalent system that absorbs the carrier-frequency hopping into the channel impulse response (CIR). The sample-spaced low-pass-equivalent CIR for the $q$-th band is given by

$$
h_{q}(n)=X \sum_{l=0}^{L_{h}} \sum_{k=0}^{K} \alpha_{k, l} e^{-j 2 \pi f_{q}\left(T_{l}+\tau_{k, l}\right)} p\left(n T-T_{l}-\tau_{k, l}-t_{0}\right)
$$

where the effect of the combined transmit and receive filter with the impulse response $p(t)$ has been included in the CIR and $t_{0}$ is a delay for the causality.

Define $\left\{t_{l}^{q}\right\}=\left\{t_{l}^{q}(i): i=0, \ldots, N^{\prime}-1 ; l=1, \ldots, L_{q}\right\}$ where $\left\{t_{l}^{q}(i): i=0,1, \ldots, N^{\prime}-1\right\}$ denotes the time-domain sample index set corresponding to the $l$-th non-zero preamble symbol period in the $q$-th band with $N \leq N^{\prime} \leq N+N_{\text {pre }} . L_{q}$ is the number of nonzero preamble symbols in the $q$-th band and depends on the preamble pattern and the band index $q$. Let $\left\{x_{q}(k)\right\}$ denote the low-pass-equivalent time-domain channel output signal samples corresponding to the $q$-th band. Then the corresponding low-pass-equivalent time-domain received samples $\left\{r_{q}\left(t_{l}^{q}(i)\right)\right\}$ in the $q$-th band can be expressed as

$$
r_{q}\left(t_{l}^{q}(i)\right)=e^{j \varphi} e^{j 2 \pi v_{q} t_{l}^{q}(i) / N} x_{q}\left(t_{l}^{q}(i)\right)+n\left(t_{l}^{q}(i)\right)
$$

where $\varphi$ is an arbitrary carrier phase, $\left\{n\left(t_{l}^{q}(i)\right)\right\}$ are zero-mean independent and identically distributed circularlysymmetric complex Gaussian noise samples with variance $\sigma^{2}=E\left\{\left|n\left(t_{l}^{q}(i)\right)\right|^{2}\right\}$, and $v_{q}$ is the normalized CFO of the $q$-th band which is related to the normalized OFO $v$ by

$$
v_{q}=b_{q} v, \text { for } q=1,2,3 .
$$

For the MB-OFDM carrier frequency synthesizer in [2], we have $\left[b_{1}, b_{2}, b_{3}\right]=\left[\frac{13}{16}, \frac{15}{16}, \frac{17}{16}\right]$ (c.f. [15]).

\section{Proposed Synchronization Method}

In this paper, we consider three tasks - sync detection, timing offset estimation and OFO estimation for the packetbased MB-OFDM system in [3]. Since the preambles in [3] possess periodicity due to identical OFDM symbols, we utilize this periodicity and develop a low complexity synchronization method while taking into account the distinctive features of the MB-OFDM system. We assume that the receiver knows what preamble the transmitter uses but not the OFO and the arrival time of the preamble.

The whole interval of the 21 preamble symbols with the sample index set $\left\{t_{l}^{q}(i)\right\}$ is divided into three disjoint parts - part- $a$, part- $b$, and part- $c$ - with the corresponding sample index sets $\left\{t_{a, l}^{q}(i)\right\},\left\{t_{b, l}^{q}(i)\right\}$, and $\left\{t_{c, l}^{q}(i)\right\}$, respectively. All the distances of the preamble symbol pairs within part$x$ are denoted by $\left\{d_{x}(m): m=1,2, \ldots\right\}$ where $x$ is $a, b$, or $c$ representing the corresponding part, or $x$ is $b c$ representing the combined interval of part- $b$ and part- $c$. For example, for the preamble pattern 1, we may set the first part as 6 symbol interval, and the second part as 12 symbol interval (see Fig. 1). In this case, $\left\{t_{a, l}^{q}(i)\right\}=\left\{t_{l}^{q}(i)\right.$ : $l=1,2\},\left\{t_{b, l}^{q}(i)\right\}=\left\{t_{l}^{q}(i): l=3,4,5,6\right\},\left\{t_{c, l}^{q}(i)\right\}$ $=\left\{t_{7}^{q}(i)\right\},\left\{d_{b}(m)\right\}=\left\{3 M_{0}, 6 M_{0}, 9 M_{0}\right\}$, and $\left\{d_{b c}(m)\right\}$ $=\left\{3 M_{0}, 6 M_{0}, 9 M_{0}, 12 M_{0}\right\}$. We also define $d_{x}(0)=0$ for convenience.
Define the correlation term with a correlation distance $d_{x}(m)$ between non-zero received preamble symbols within part- $x$ in the $q$-th band as

$$
R_{x, q}\left(m, k_{\mathrm{t}}, N^{\prime}\right)=\sum_{\substack{\left\{k, k+d_{x}(m)\right\} \in\left\{k_{\mathrm{t}}+\\ t_{x, l}^{q}(i): i=0,1, \ldots, N^{\prime}-1\right\}}} \tilde{r}_{q}^{*}(k) \tilde{r}_{q}\left(k+d_{x}(m)\right)
$$

where $k_{t}$ is the reference timing index. During part- $a$, where the sync detection and coarse timing estimation are performed, the receiver carrier frequency hopping is not performed. During the remaining parts, the receiver carrier frequency is hopped according to the preamble pattern. The fine timing estimation is based on the received preamble symbols within part-b while the OFO (and also channel) estimation is based on both part-b and part-c. The reason for the use of only part-b for the fine timing estimation is to allow processing time so that timing adjustment (for frequency hopping instants) based on the fine timing estimation can be made at the end of the preamble.

\section{A. Sync Detection and Coarse Timing Estimation}

The receiver's carrier frequency is initially set to the one that corresponds to the first preamble symbol, and denote the corresponding band index by $\bar{q}$. During the sync detection process, receiver carrier frequency hopping is not performed. Denote the corresponding low-pass equivalent received samples by $\{\tilde{r}(k)\}$. The initial part of $\{\tilde{r}(k)\}$ contains noise-only samples followed by the beginning of the received preamble. The number of the initial noise-only samples is a random variable depending on the arrival time of the preamble and the receiver's sync detection window timing. We use the first two preamble symbols in the $\bar{q}$ th band to detect the arrival of the preamble. Then the sync detection metric is given by

$$
\mathcal{M}(k)=\left|R_{a, \bar{q}}\left(1, k_{\mathrm{t}}, N^{\prime}\right)\right| .
$$

For complexity reduction and energy saving, the computation of $\mathcal{M}(k)$ is performed only at $k=0, \Delta, 2 \Delta, \ldots$, where $\Delta$ (an integer) is a design parameter.

Once $\mathcal{M}(k)$ becomes larger than a pre-defined threshold $\mathcal{M}_{\mathrm{SD}}$, say at $k=k_{\mathrm{SD}}$, the receiver decides that the preamble has been detected and starts finding the coarse timing point as follows:

$$
k_{\mathrm{ct}}=\underset{k_{\mathrm{SD}} \leq k \leq k_{\mathrm{SD}}+W_{1}-1}{\arg \max }\{\mathcal{M}(k)\}
$$

where $W_{1}$ is the length of the window within which the coarse timing point is searched for and can be set to $M_{0}$. A lower complexity version of the above coarse timing estimator can be implemented by finding the maximum point, say $k_{1}$, of the metric values at $k=k_{\mathrm{SD}}, k_{\mathrm{SD}}+\Delta_{1}, k_{\mathrm{SD}}+2 \Delta_{1}, \ldots$, and then the maximum point of the metric values at $k_{1}-\left\lceil\Delta_{1} / 2\right\rceil$, $k_{1}-\left\lceil\Delta_{1} / 2\right\rceil+1, \ldots, k_{1}+\left\lceil\Delta_{1} / 2\right\rceil$. Based on the coarse timing point, the receiver determines at what time instant it should hop the carrier frequency to demodulate the rest of the preamble. The probability of missed detection and false alarm for a given $\mathcal{M}_{\mathrm{SD}}$ is derived in Appendix $\mathrm{B}$.

\section{B. Fine Timing Offset Estimation}

The fine timing estimation is given by

$$
k_{\mathrm{ft}}=\underset{i \in\left(k_{\mathrm{ct}}-W_{2}, k_{\mathrm{ct}}+W_{3}\right)}{\arg \max } \sum_{q=1}^{3}\left|R_{b, q}\left(1, i, N^{\prime}\right)\right|
$$


where the trial fine timing points consist of the coarse timing point, $W_{2}$ sample points before and $W_{3}$ sample points after the coarse timing point. Note that although more correlation terms can be included in the fine timing estimation, for low complexity implementation we just use the correlation terms with the correlation distance $d_{b}(1)$. The correlation window length $N^{\prime}$ of each preamble symbol will be optimized to achieve better timing estimation.

\section{OFO Estimation}

We adopt the OFO estimator from [15] which is based on the BLUE principle and the correlations among the nonzero received preamble symbols within the same band. The differences are as follows. In [15] the OFO estimator utilizes all preamble symbols, while in this paper the OFO estimator is based only on part-b and part-c allowing processing time and resources for sync detection and coarse timing estimation. Furthermore, the correlation window length for each preamble symbol is fixed at $N$ samples in [15] but is set to $N^{\prime}$ in this paper which will be optimized for better estimation performance.

The OFO estimator is given by

$$
\widehat{v}=\frac{\sum_{q=1}^{3} \mathbf{1}^{T} C_{\theta_{q}}^{-1} \mathbf{1} b_{q} \widehat{v}_{q}}{\sum_{q=1}^{3} \mathbf{1}^{T} C_{\theta_{q}}^{-1} \mathbf{1} b_{q}^{2}}
$$

where

$$
\begin{aligned}
\widehat{v}_{q} & =\sum_{m=1}^{H_{q}} \omega_{q}(m) \theta_{q}(m) \\
\theta_{q}(m) & =\frac{N}{2 \pi d_{\mathrm{bc}}(m)} \operatorname{angle}\left\{R_{b c, q}\left(m, k_{\mathrm{ft}}, N^{\prime}\right)\right\}
\end{aligned}
$$

with $\omega_{q}(m)$ being the $m$-th component of the weighting vector

$$
\boldsymbol{\omega}_{q}=C_{\theta_{q}}^{-1} \mathbf{1}\left(\mathbf{1}^{T} C_{\theta_{q}}^{-1} \mathbf{1}\right)^{-1}
$$

In (13), $\boldsymbol{C}_{\theta_{q}}$ is the covariance matrix of $\left\{\theta_{q}(m): m=\right.$ $\left.1, \ldots, H_{q}\right\}$ and 1 denotes the all-one column vector, whose size should be apparent from the expression. $H_{q}$ is the design parameter of the BLUE method for the $q$-th band. Two approaches (Methods A and B) for the calculation of $\left\{\boldsymbol{C}_{\theta_{q}}\right\}$ are given in [15]. Due to different window length in this paper, the covariance matrices $\left\{\boldsymbol{C}_{\theta_{q}}\right\}$ become slightly different from those in [15]. For example, for preamble pattern 1 or 2 , denoting the number of non-zero preamble symbols within the combined part- $b$ and part- $c$ in the $q$ th band by $\bar{L}_{q}$, and applying Method A, we obtain

$$
\begin{aligned}
\boldsymbol{C}_{\theta_{q}}(m, n)= & \frac{N^{2} \sigma^{2}}{4 \pi^{2}\left(3 M_{0}\right)^{2} E_{x, q}\left(N^{\prime}\right)} \frac{1}{m n\left(\bar{L}_{q}-m\right)\left(\bar{L}_{q}-n\right)} \\
& \times \begin{cases}m+\frac{\left(\bar{L}_{q}-m\right) N^{\prime} \sigma^{2}}{2 E_{x, q}\left(N^{\prime}\right)}, & \text { if } m=n \& m<\bar{L}_{q} / 2 \\
\left(\bar{L}_{q}-m\right)+\frac{\left(\bar{L}_{q}-m\right) N^{\prime} \sigma^{2}}{2 E_{x, q}\left(N^{\prime}\right)}, & \text { if } m=n \& m \geq \bar{L}_{q} / 2 \\
\min (m, n), & \text { if } m \neq n \& m+n<\bar{L}_{q} \\
\bar{L}_{q}-\max (m, n), & \text { if } m \neq n \& m+n \geq \bar{L}_{q}\end{cases}
\end{aligned}
$$

and applying Method B gives

$$
\begin{aligned}
\boldsymbol{C}_{\theta_{q}}(m, n) & =\frac{N^{2} \sigma^{2}}{4 \pi^{2}\left(3 M_{0}\right)^{2} E_{x, q}\left(N^{\prime}\right)} \frac{1}{m n\left(\bar{L}_{q}-m\right)\left(\bar{L}_{q}-n\right)} \\
& \times \begin{cases}\min (m, n), & \text { if } m+n<\bar{L}_{q} \\
\bar{L}_{q}-\max (m, n), & \text { if } m+n \geq \bar{L}_{q}\end{cases}
\end{aligned}
$$

where $E_{x, q}\left(N^{\prime}\right)$ is the received preamble symbol energy contained in $N^{\prime}$ samples in the $q$ th band. The estimator in (10) can be implemented as

$$
\widehat{v}=\frac{\sum_{q=1}^{3} \widehat{E}_{x, q}\left(N^{\prime}\right) A_{q} b_{q} \widehat{v}_{q}}{\sum_{q=1}^{3} \widehat{E}_{x, q}\left(N^{\prime}\right) A_{q} b_{q}^{2}}
$$

where the estimate of $\widehat{E}_{x, q}\left(N^{\prime}\right)$ is calculated as

$$
\widehat{E}_{x, q}\left(N^{\prime}\right)=R_{b c, q}\left(0, k_{\mathrm{ft}}, N^{\prime}\right) / \bar{L}_{q},
$$

and

$$
A_{q}=\frac{\mathbf{1}^{T} \boldsymbol{C}_{\theta_{q}}^{-1} \mathbf{1}}{E_{x, q}\left(N^{\prime}\right)}
$$

\section{Optimizing the CoRrelation Window Lengths}

We consider the combined interval of $N_{\text {suf }}$ zero-amplitude suffix samples from the $i$ th symbol and $N_{\text {pre }}$ zero-amplitude prefix samples from the $i+1$ th symbol as the $N_{\text {pre }}$ zeroamplitude suffix samples from the $i$ th symbol and $N_{\text {suf }}$ zeroamplitude prefix samples from the $(i+1)$ th symbol. We assume that the maximum channel dispersion is $N_{\text {pre }}=32$ samples, and the interval of the $N_{\text {suf }}=5$ prefix samples is reserved for switching the carrier frequency. Hence, the considered correlation window length range is $\left[N, N+N_{\text {pre }}\right]=[128,160]$.

\section{A. Window Length for Timing Estimation}

\section{Define}

$$
\beta\left(i, T_{1}, N^{\prime}\right)=R_{a, 1}\left(1, T_{1}+i+1, N^{\prime}\right)-R_{a, 1}\left(1, T_{1}+i, N^{\prime}\right)
$$

Then for a given channel output signal $\left\{x_{1}(k)\right\}, \beta\left(i, T_{1}, N^{\prime}\right)$ are independent complex Gaussian random variables with

$$
\begin{aligned}
E\left[\beta\left(i, T_{1}, N^{\prime}\right)\right] & =x_{1}^{*}\left(i+N^{\prime}\right) x_{1}\left(i+N^{\prime}+d_{a}(1)\right)-x_{1}^{*}(i) x_{1}\left(i+d_{a}(1)\right) \\
\operatorname{Var}\left[\beta\left(i, T_{1}, N^{\prime}\right)\right] & =\sigma^{2}\left(\left|x_{1}(i)\right|^{2}+\left|x_{1}\left(i+d_{a}(1)\right)\right|^{2}\right. \\
& \left.+\left|x_{1}\left(i+N^{\prime}\right)\right|^{2}+\left|x_{1}\left(i+N^{\prime}+d_{a}(1)\right)\right|^{2}\right)+2 \sigma^{4} .
\end{aligned}
$$

Then we use the following criterion to design the correlation window length $N_{t}$ for timing estimation

$$
N_{t}=\underset{N \leq N^{\prime} \leq N+N_{g}}{\arg \max }\left\{\prod_{i=0}^{N / 2}\left(1-Q\left(\frac{\left|E\left[\beta\left(i, T_{1}, N^{\prime}\right)\right]\right|}{\sqrt{\operatorname{Var}\left[\beta\left(i, T_{1}, N^{\prime}\right)\right]}}\right)\right)\right\}_{(22)}
$$

where $Q(\cdot)$ is the Gaussian tail probability, and $T_{1}$ for each $N^{\prime}$ is chosen such that $\left[T_{1}, T_{1}+N^{\prime}+N / 2-1\right]$ is approximately symmetric with respect to $\left[k_{\text {ref }}, k_{\text {ref }}+N+N_{\text {pre }}\right]$. This criterion can be approximately considered as the probability of the joint events $\left\{\left|R_{a, 1}\left(1, \bar{k}, N^{\prime}\right)\right|>\left|R_{a, 1}\left(1, \bar{k}+l, N^{\prime}\right)\right|: l=\right.$ $\pm 1, \pm 2, \ldots\}$ where $\bar{k}$ is the time instant corresponding to the maximum of $\left|R_{a, 1}\left(1, k, N^{\prime}\right)\right|$. In the design, we replace $\left|x_{1}(k)\right|^{2}$ with $E\left[\left|x_{1}(k)\right|^{2}\right]$ which can be calculated using the channel power delay profile $\left\{\delta_{i}^{2}\right\}$ obtained in the Appendix A. 


\section{B. Window Length for OFO Estimation}

We use the BLUE variance of the CFO estimation to optimize the window length for our OFO estimator. We can simply consider the BLUE variance in the $q$ th band given by

$$
\operatorname{Var}\left\{\widehat{v}_{q} \mid N^{\prime}\right\}=\left(\mathbf{1}^{T} \boldsymbol{C}_{\theta_{q} \mid N^{\prime}}^{-1} \mathbf{1}\right)^{-1}
$$

where $\boldsymbol{C}_{\theta_{q} \mid N^{\prime}}$ is given in (14). Then the best window length $N_{f}$ for our OFO estimator is determined by

$$
N_{f}=\underset{N \leq N^{\prime} \leq N+N_{\text {pre }}}{\arg \min }\left\{\operatorname{Var}\left\{\widehat{v}_{q} \mid N^{\prime}\right\}\right\} .
$$

\section{Simulation Results and Discussions}

We use the simulation parameters as specified in [3]: $N=$ $128, N_{\text {pre }}=32, N_{\text {suf }}=5, N_{g}=37$, carrier frequencies $f_{1}=3432 \mathrm{MHz}, f_{2}=3960 \mathrm{MHz}, f_{3}=4488 \mathrm{MHz}$, the sub-carrier spacing $1 / T=4.125 \mathrm{MHz}$, and preamble pattern $1 .^{1}$ The normalized OFO $v$ is set to 0.02 . The channel model CM-2 with $L_{h}=6$ and $K=6$ is adopted. $p(t)$ is a spectral raised cosine pulse with a filter span of $\left[-5 T_{s}, 5 T_{s}\right]$ and $t_{0}=$ $5 T_{s}$. The parameters of the synchronization method used are $\mathcal{M}_{\mathrm{SD}}=0.5 N \sigma^{2}, \Delta=8, \Delta_{1}=1, W_{1}=165, W_{2}=32$, $W_{3}=32$, and $H_{q}=2$.

Fig. 2 shows the proposed metric of the timing correlation window length design. For all SNRs of 5, 10, and $15 \mathrm{~dB}$, the timing correlation window length of $N^{\prime}=132$ gives the best design metric. The metric differences are very small for $128 \leq$ $N^{\prime} \leq 136$, but become significant as $N^{\prime}$ increases further. The design results are insensitive to SNR values. The simulation results of the fine timing estimation obtained with different window lengths are plotted in Fig. 3 in terms of the mean and the variance of the timing offset estimate. The mean of the timing offset estimate ${ }^{2}$ depends on the correlation window length which can be ascribed to the different energy captures of different window sizes. The timing offset estimation variances of different window sizes match very well with our design metric in Fig. 2.

The design metric (BLUE variance) of the CFO correlation window length is shown in Fig. 4. The plots for different SNR values are normalized and scaled by $\mathrm{SNR}^{1 / 100}$ for convenience in presentation. The results in the left figure are obtained with a fixed timing point at $k_{\text {ref }}$ while those in the right figure correspond to using the best timing point which gives the smallest BLUE variance for each correlation window length. The minimum BLUE variances for SNR of 5, 10, and 15 $\mathrm{dB}$ are achieved at $N^{\prime}=141,143$, and 144, respectively in the left figure, and at $N^{\prime}=135,138$, and 139, in the right figure. The larger SNR tends to yield a slightly larger $N^{\prime}$. However, it should be mentioned that the BLUE variance differences of different $N^{\prime}$ are extremely small, and hence in practice a smaller fixed $N^{\prime}$ (say 132 which is the same as the correlation window length for timing) may be used for all SNR which has slightly lower complexity than $N^{\prime}$ values with minimum BLUE variance. In Fig. 5, we present simulation

\footnotetext{
${ }^{1}$ Our method can also be applied to other preamble patterns.

${ }^{2}$ It can be shifted by a timing offset advancement as described in [12].
}

results for the OFO estimation MSEs obtained with different $N^{\prime}$ values. Different window sizes give virtually the same MSE performance. Hence, using the best timing correlation window length for the CFO correlation window length is a good design choice.

Fig. 6 compares the fine timing estimation results obtained with different values of design parameters $\Delta$ and $\Delta_{1}$. The simulation results show that the timing estimators with three sets of design parameters $\left(\Delta=1, \Delta_{1}=1\right),\left(\Delta=8, \Delta_{1}=1\right)$ and ( $\left.\Delta=16, \Delta_{1}=4\right)$ yield almost the same performance. This illustrates that proper choice of parameters in the proposed method (say $\Delta=16$ and $\Delta_{1}=4$ ) gives significant complexity saving while achieving virtually the same performance. Note that conventional methods use $\Delta=1$ and $\Delta_{1}=1$. By using $\Delta=16$ and $\Delta_{1}=4$, the proposed method gives complexity reduction of approximately a factor of 16 in the sync detection and a factor of 4 in the coarse timing estimation, if compared to conventional methods.

\section{CONCLUSIONS}

We have presented a low complexity synchronization method for MB-OFDM based UWB systems which utilizes the distinctive features of MB-OFDM systems and takes into account the interplay between the timing and carrier frequency hopping at the receiver. We have derived the probability density functions (pdfs) of the delay of the UWB channel paths which are used in optimizing our synchronization method. These pdfs are also useful in evaluating or optimizing the energy capture of other UWB systems. The timing estimation performance is more sensitive to the correlation window length than the frequency offset estimation. Hence, although the best correlation window lengths for timing and frequency offset estimation are not the same, we may use the same correlation window length designed for the timing estimation when performing both tasks. The simulation results corroborate the effectiveness of our proposed synchronization method and optimization criteria.

\section{Appendix A: AVERAGE ENERGY-CAPture}

There is no difference of average energy capture within the same window length among different bands, and hence for simplicity we drop the band index $q$ in the following. Let $L^{\prime}$ denote the maximum length of the sample-spaced CIR vector and $\boldsymbol{h}$ denote the length $L^{\prime} \times 1$ CIR vector. Depending on the actual length of each channel realization, $\boldsymbol{h}$ may contain some zero samples. Then the energy of the $N^{\prime} \times 1$ channel output vector $\boldsymbol{x}$ can be given by

$$
E_{x}\left(N^{\prime}\right) \triangleq E\left[\boldsymbol{x}^{H} \boldsymbol{x}\right]=\operatorname{Trace}\left[\boldsymbol{S} \boldsymbol{C}_{h} \boldsymbol{S}^{H}\right]
$$

where $\boldsymbol{S}$ is the $N^{\prime} \times L^{\prime}$ transmitted signal matrix with $[\boldsymbol{S}]_{k, l}=$ $s(k-l)$ and $\boldsymbol{C}_{h}$ is the covariance matrix of $\boldsymbol{h}$. For simplicity in the design, we assume that $\boldsymbol{C}_{h}=\operatorname{diag}\left\{\delta_{0}^{2}, \delta_{1}^{2}, \ldots, \delta_{L^{\prime}-1}^{2}\right\}$. Then from (3) we can calculate $\delta_{i}^{2}$ as $^{3}$

$$
\begin{aligned}
\delta_{i}^{2} & =\int_{0}^{\infty} \int_{0}^{\infty} \sum_{l=0}^{L_{h}} \sum_{k=0}^{K} E\left[\left|\alpha_{k, l}\left(T_{l}, \tau_{k, l}\right)\right|^{2}\right] p^{2}\left(i T_{s}-T_{l}-\tau_{k, l}-t_{0}\right) \\
& \times f_{T_{l}, \tau_{k, l}}\left(T_{l}, \tau_{k, l}\right) d T_{l} d \tau_{k, l}
\end{aligned}
$$

${ }^{3}$ We neglect the shadowing parameter $X$ in this paper. 
where we have used the statistical independence among $\alpha_{k, l}\left(T_{l}, \tau_{k, l}\right)$. The required joint probability density function (pdf) of $T_{l}$ and $\tau_{k, l}$ is pursued below in a more general framework of the pdf of the delay $Z_{k, l} \triangleq T_{l}+\tau_{k, l}$ for the $k$-th ray of the $l$-th cluster of the UWB channel.

Note that $Z_{k, l}$ can be expressed as the sum of inter-arrival times of the first $l$ clusters and the first $k$ rays. Since interarrival times of the UWB channel paths are independent, $Z_{k, l}$ is the sum of $(l+k-2)$ independent exponential random variables. From the characteristic function of $Z_{k, l}$, we obtain the pdf of $Z_{k, l}$ as ${ }^{4}$

$$
f_{Z_{k, l}}\left(z_{k, l}\right)= \begin{cases}\left(R_{1}+R_{2}\right) \Lambda^{l} \lambda^{k}(-1)^{(l+k)} & k \neq 0 \text { or } l \neq 0 \\ \delta\left(z_{k, l}\right) & k=0 \& l=0\end{cases}
$$

where

$$
\begin{aligned}
& R_{1}=\left\{\begin{array}{cl}
(-1)^{l} e^{-\Lambda z_{k, l}} \sum_{i=0}^{l-1} \frac{\prod_{m=0}^{i-1}(k+m)}{i ! l-i-1) !} & k \neq 0 \& l \neq 0 \\
\times(\Lambda-\lambda)^{(-k-i)} z_{k, l}^{(-i-1)} u\left(z_{k, l}\right) & k=0 \& l \neq 0 \\
\frac{(-1)^{l}}{(l-1) !} z_{k, l}^{(l-1)} e^{-\Lambda z_{k, l}} u\left(z_{k, l}\right) & l=0 \\
0 &
\end{array}\right. \\
& R_{2}=\left\{\begin{array}{cl}
(-1)^{k} e^{-\lambda z_{k, l} \sum_{i=0}^{k-1} \frac{\prod_{m=0}^{i-1}(l+m)}{i !(k-i-1) !}} & k \neq 0 \& l \neq 0 \\
\times(\Lambda-\lambda)^{(-l-i)} z_{k, l}^{(k-i-1)} u\left(z_{k, l}\right) & k \neq 0 \& l=0 \\
\frac{(-1)^{k}}{(l-1) !} z_{k, l}^{(k-1)} e^{-\lambda z_{k, l} u\left(z_{k, l}\right)} & k=0, \\
0
\end{array}\right.
\end{aligned}
$$

$\Lambda$ and $\lambda$ are the cluster arrival rate and the ray arrival rate, respectively, and $u(\cdot)$ is the unit step function.

Since $\left\{T_{l}\right\}$ and $\left\{\tau_{k, l}\right\}$ are independent, we have

$$
f_{T_{l}, \tau_{k, l}}(x, y)=f_{t_{l}}(x) f_{\tau_{k, l}}(y)
$$

where the pdfs of $T_{l}$ and $\tau_{k, l}$ are related to the pdf of $Z_{k, l}$ as

$$
\begin{aligned}
f_{T_{l}}(x) & =f_{Z_{0, l}}(x) \\
f_{\tau_{k, l}}(y) & =f_{Z_{k, 0}}(y) .
\end{aligned}
$$

Substituting (27)-(32) and (2) into (26) gives

$$
\begin{aligned}
\delta_{i}^{2} & =\sum_{l=0}^{L_{h}} \sum_{k=0}^{K} \int_{0}^{T} \int_{0}^{T} \Omega_{0} e^{-T_{l} / \Gamma} e^{-\tau_{k, l} / \gamma} p^{2}\left(i T_{s}-T_{l}-\tau_{k, l}-t_{0}\right) \\
& \times f_{Z_{0}, l}\left(T_{l}\right) f_{Z_{k, 0}}\left(\tau_{k, l}\right) d T_{l} d \tau_{k, l}
\end{aligned}
$$

which can be numerically evaluated. Then from (25) we immediately obtain $E_{x}\left(N^{\prime}\right)$. We normalize $\left\{\delta_{i}^{2}\right\}$ to give $\sum_{i=0}^{L^{\prime}} \delta_{i}^{2}=1$.

\section{ApPendix B: The Probability of False Alarm AND Missed Detection of The SynC Detection}

Denote the sync detection metric $\mathcal{M}(k)$ by $Z$. When the receiver input corresponds to part-a of the preamble, given the channel response, $Z$ has approximately a Rician pdf as

$$
f_{Z}^{(1)}(z)=\frac{1}{\sigma_{1}^{2}} z e^{\frac{-\left(z^{2}+\mu^{2}\right)}{2 \sigma_{1}^{2}}} I_{0}\left(\frac{z \mu}{\sigma_{1}^{2}}\right)
$$

where $\mu=E_{x}\left(N^{\prime}\right)$ and $\sigma_{1}^{2}=E_{x}\left(N^{\prime}\right) \sigma^{2}+\frac{1}{2} N^{\prime} \sigma^{4}$. When the receiver input contains noise only, $Z$ has approximately a Rayleigh pdf as

$$
f_{Z}^{(2)}(z)=\frac{1}{\sigma_{2}^{2}} z e^{\frac{-z^{2}}{2 \sigma_{2}^{2}}}
$$

\footnotetext{
${ }^{4}$ Detailed steps are skipped due to space limitation.
}

where $\sigma_{2}^{2}=\frac{1}{2} N^{\prime} \sigma^{2}$. Then the probability of false alarm $P F A$ and the probability of missed detection $P M D$ for a given $\tau=\mathcal{M}_{\mathrm{SD}}$ can be expressed as

$$
\begin{array}{cl} 
& P F A_{z \mid \tau}(z \mid \tau)=\int_{\tau}^{\infty} f_{Z}^{(2)}(z) d z=e^{\frac{-\tau^{2}}{2 \sigma_{2}^{2}}} \\
& P M D_{z \mid \tau}(z \mid \tau)=\int_{-\infty}^{\infty} P M D_{z \mid \tau, E}(z \mid \tau, E) f_{E}(E) d E \\
\text { where } \quad & P M D_{z \mid \tau, E}(z \mid \tau, E)=\int_{0}^{\tau} f_{Z}^{(1)}(z) d z \\
& f_{E}(E)=E^{\frac{a}{2}-1} e^{-\frac{E}{2 b^{2}}} /\left(b^{a} 2^{\frac{a}{2} \Gamma\left(\frac{a}{2}\right)}\right)
\end{array}
$$

Note that the exact pdf of $E$ (the channel output preamble symbol energy) for the UWB channel model in [16] is intractable, but it can be well approximated by a Gamma pdf as given in (39), where the parameter $a=6.4$ and $b^{2}=\frac{20.8 E_{x}\left(N^{\prime}\right)}{N^{\prime}}$ are obtained by simulation.

\section{REFERENCES}

[1] A. Batra, et. al., "Multi-band OFDM Physical Layer Proposal for IEEE 802.15 Task Group 3a," IEEE P802.15-03/268r3, Orlando, FL, USA, Mar. 2004.

[2] A. Batra, J. Balakrishnan, G. R. Aiello, J. R. Foerster, and A. Dabak, "Design of a multiband OFDM system for realistic UWB channel environments," IEEE Trans. Microwave Theory and Techniques, Sept. 2004, pp. 2123-2137.

[3] IEEE 802.15 Working Group for Wireless Personal Area Networks (WPANs)' "Multi-band OFDM Physical Layer Proposal for IEEE 802.15 Task Group 3a," Mar. 2004.

[4] D. Dardali, V. Tralli, and A. Vaccari, "A theoretical characterization of nonlinear distortion effects in OFDM systems," IEEE Trans. Commun., vol. 48 , no. 10 , Oct. 2000 , pp. $1755-1764$.

[5] T. Pollet, M. Van Bladel, and M. Moeneclaey, "BER sensitivity of OFDM systems to carrier frequency offset and Wiener phase noise," IEEE Trans. Commun., vol. 43, pp. 191-193, Feb./Mar./Apr. 1995.

[6] H. Steendam and M. Moeneclaey, "Synchronization sensitivity of multicarrier systems," European Communications, ETT special issue on MultiCarrier Spread Spectrum, vol. 52, no. 5, pp. 834-844, May 2004.

[7] P. H. Moose, "A technique for orthogonal frequency division multiplexing frequency offset correction," IEEE Trans. Commun., Oct. 1994, pp. 29082914.

[8] T. M. Schmidl and D. C. Cox, "Robust frequency and timing synchronization for OFDM," IEEE Trans. Commun., Dec. 1997, pp. 1614-1621.

[9] M. Morelli and U. Mengali, "An improved frequency offset estimator for OFDM applications," IEEE Commun. Letters, Mar. 1999, pp. 75-77.

[10] T. Keller, L. Piazzo, P. Mandarini, and L. Hanzo, "Orthogonal frequency division multiplex synchronization techniques for frequency-selective fading channels," IEEE J. Select. Areas Commun., vol. 19, pp. 999-1008, June 2001.

[11] A. J. Coulson," "Maximum likelihood synchronization for OFDM using a pilot symbol: Algorithms," IEEE JSAC, vol. 19, no. 12, pp. 2486-2494, Dec. 2001.

[12] H. Minn, V. K. Bhargava and K. B. Letaief, "A robust timing and frequency synchronization for OFDM systems, IEEE Trans. Wireless Commun., vol. 2, no. 4, July 2003, pp. 822-839.

[13] H. Minn, V. K. Bhargava, and K. B. Letaief, "A combined timing and frequency synchronization and channel estimation for OFDM, IEEE Trans. Commun., vol. 54, no. 3, pp. 416-422, Mar. 2006.

[14] H. Minn and P. Tarasak, "Improved maximum likelihood frequency offset estimation based on likelihood metric design," IEEE Trans. Signal Processing, vol. 54, no. 6, pp. 2076-2086, June 2006.

[15] Y. Li, T. Jacobs, and H. Minn, "Frequency offset estimation for MBOFDM-based UWB systems," IEEE ICC'06, June 2006.

[16] A. F. Molisch, J. R. Foerster, M. Pendergrass, "Channel models for ultrawideband personal area networks," IEEE Wireless Communications Magazine, vol. 10, no. 6, pp. 14-21, Dec. 2003.

[17] M. Z. Win and R. A. Scholtz, "On the energy capture of ultra-wide bandwidth signals in dense multipath environments," IEEE Commun. Lett., vol. 2, no. 9, pp. 245-247, Sept. 1998. 


\section{ACKNOWLEDGMENT}

This work is supported by the Erik Josson School Research Excellence Initiative, UTD.

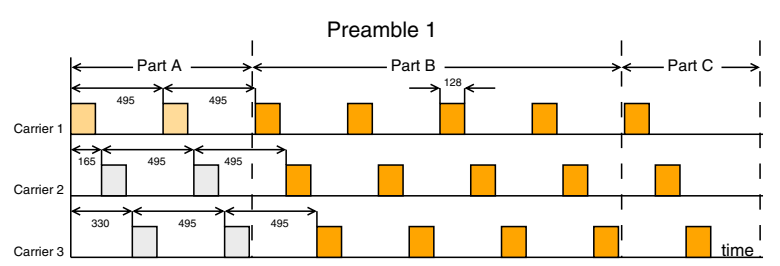

Fig. 1. The structure of the preamble pattern 1 from [3] (Also shown are the three preamble parts used in our synchronization method. The preamble symbols of carrier 2 and 3 within Part-A are not used in our synchronization method).

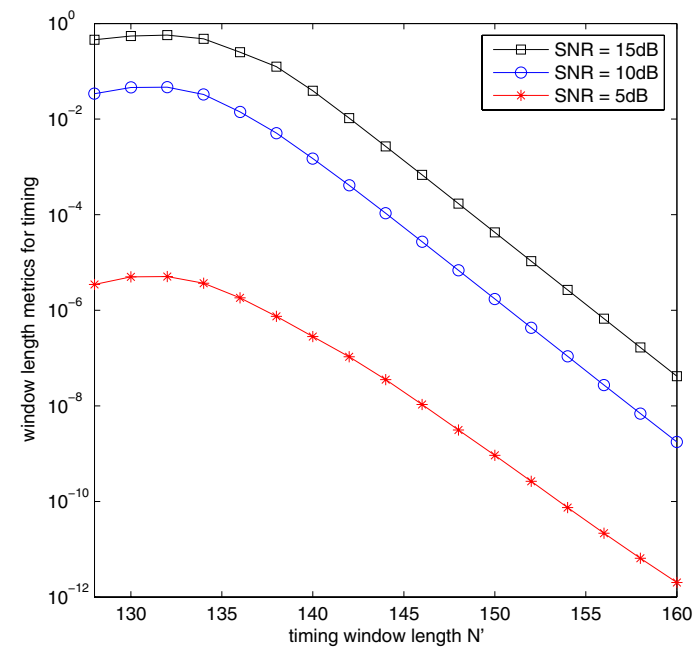

Fig. 2. The timing correlation window length design metric
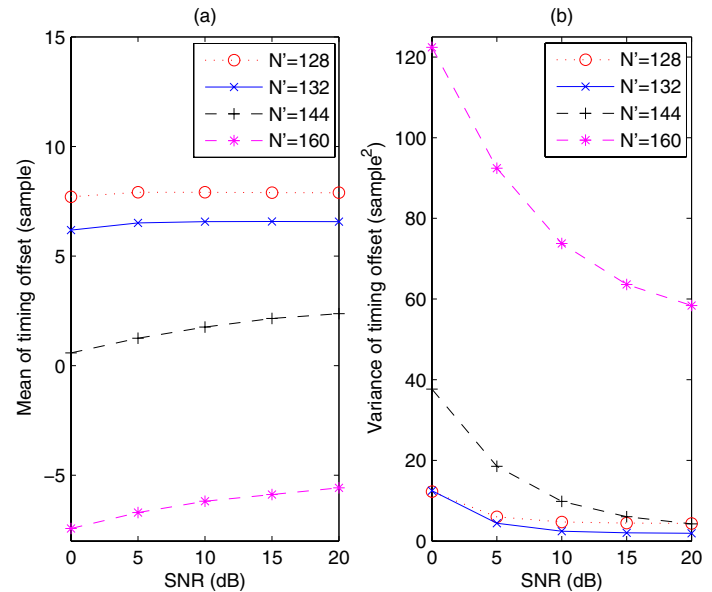

Fig. 3. The simulation results of the fine timing offset estimation
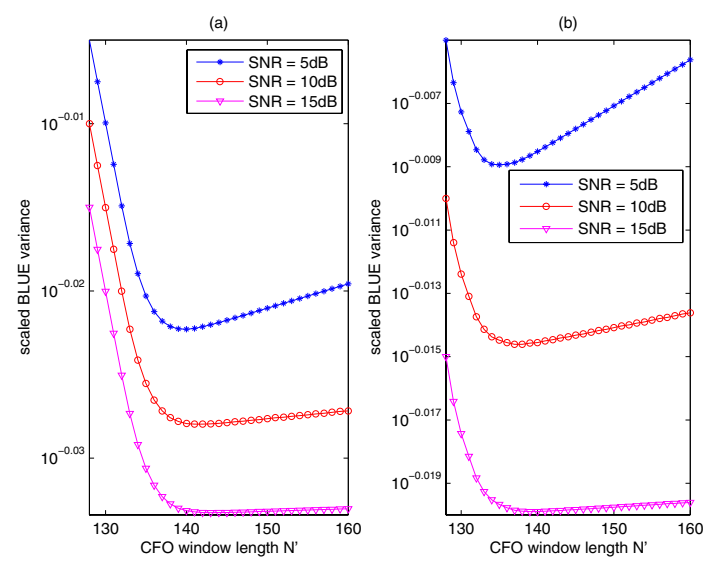

Fig. 4. The CFO estimation correlation window length design metric

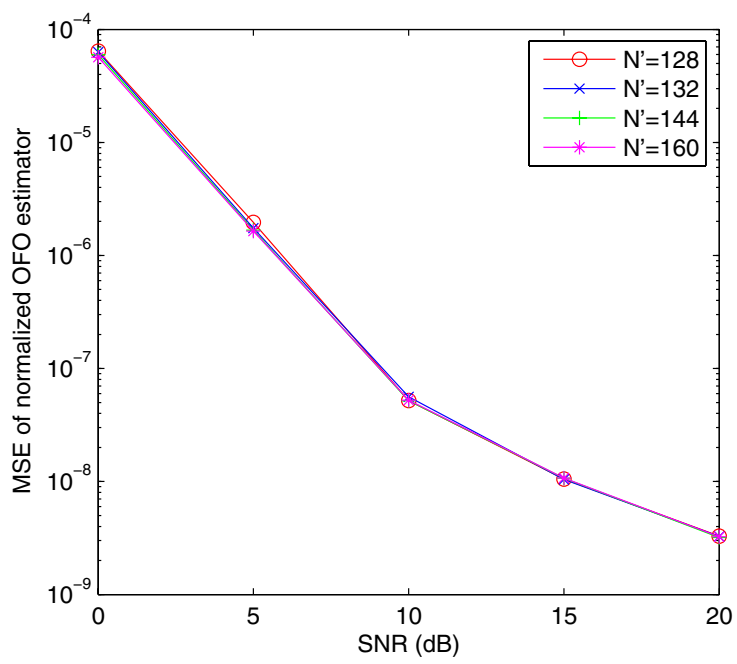

Fig. 5. The simulation results of the $\mathrm{OFO}$ estimation
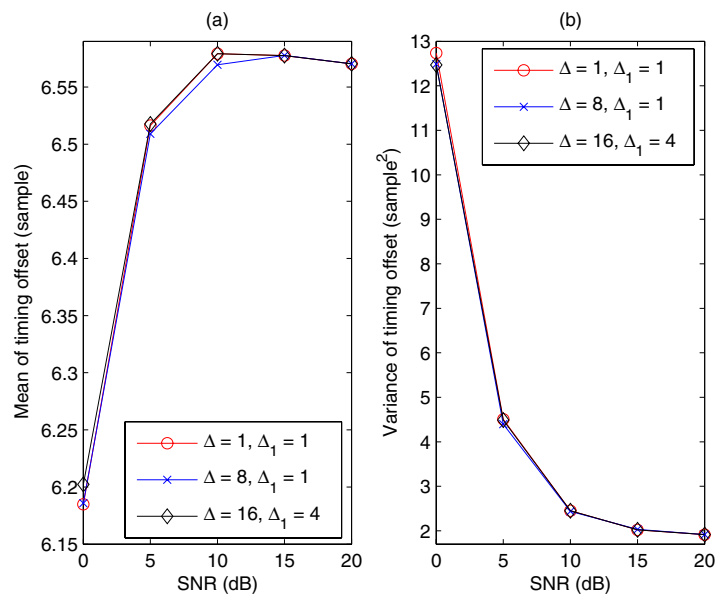

Fig. 6. The simulation results of the fine timing offset estimation for different values of design parameters $\Delta$ and $\Delta_{1}$ 